\title{
STRIKE3-Case Study for Standardized Testing of Timing-grade GNSS Receivers against Real-world Interference Threats
}

\author{
Amin Hashemi \\ Finnish Geospatial Research Institute, \\ National Land Survey of Finland \\ Kirkkonummi, Finland \\ amin.hashemi@nls.fi \\ M. Zahidul H. Bhuiyan \\ Finnish Geospatial Research Institute, \\ National Land Survey of Finland \\ Kirkkonummi, Finland \\ zahidul.bhuiyan@nls.fi
}

\author{
Sarang Thombre \\ Finnish Geospatial Research Institute, \\ National Land Survey of Finland \\ Kirkkonummi, Finland \\ sarang.thombre@nls.fi
}

Michael Pattinson

Nottingham Scientific Limited

Nottingham, United Kingdom

michael.pattinson@nsl.eu.com

\author{
N. Giorgia Ferrara \\ Finnish Geospatial Research Institute, \\ National Land Survey \\ Kirkkonummi, Finland \\ giorgia.ferrara@nls.fi
}

\begin{abstract}
One of the major use cases of GNSS signals is precise time keeping but introduction of interference signals has threatened the reliability of such a functionality. Therefore, it is necessary to identify signal types most threatening to GNSS and adopt solutions as they evolve. We studied the performance of a timing-grade GNSS receiver in presence of interference signals. We tested the robustness of the receiver against different classes of signals which were captured in real world and identified as most threatening interference to GNSS by the EU Horizon 2020 research project STRIKE3. We showed that the quality of the time solution provided by the receiver degrades in their presence. Through statistical analysis we characterized the degradation of the solution in case of different classes of interference signals.
\end{abstract}

Keywords-Global Navigation Satellite System, Time dissemination, Interference.

\section{INTRODUCTION}

The world today is highly dependent on technologies which assure precise time keeping and reliable time dissemination. Growth of the human being as a race with an ever advancing civilization requires this even more, as without it most industries would have not been possible. The Global Navigation Satellite System (GNSS) provides such a service. A GNSS receiver is designed to compute a position, velocity and time (PVT) solution. Essentially GNSS can be used for time-keeping purposes with an accuracy of some tens of nanoseconds [1], [2], [3]. GNSS signals once received at the user segment are weak and any sort of interference, either intentional or unintentional, can degrade the quality of the PVT solution dramatically which in turn would put end users of GNSS timing services such as large scale power distribution networks, banks, etc. in danger.

In [4] and [5] standard platform and guidelines for testing of a timing-grade GNSS receiver without the presence of interference signals is presented. In [6], capability of a geodetic class GPS receiver in delivering timing solutions for time-critical applications is determined through comparison of its timing solution as opposed to an atomic clock over 24 hours measuring sessions. Therein it is concluded that the receiver was capable of permanent delivering of time marks within millisecond accuracy. Previous research work carried out in [7] analyzes the performance of a timing-grade GNSS receiver in presence of unintentionally generated in-band and out-ofband interference signals over 4 hours measuring sessions. It is shown that the time RAIM (Receiver Autonomous Integrity Monitoring) algorithm is an essential tool for enhancing the immunity of the receiver against unintentional GNSS jammer sources.

Within the scope of this case study we built on top of the previous work [8], [9], [10] which developed international standards in the specific area of GNSS threat reporting and GNSS receiver testing in the framework of H2020 European project Standardization of GNSS Threat Reporting and Receiver Testing through International Knowledge Exchange, Experimentation and Exploitation (STRIKE3). We analyzed robustness of timing-grade receivers in presence of GNSS interference signals which were identified by the STRIKE3 project as being the most threatening to GNSS in real world. The most important contribution of this work is testing of the GNSS receivers against a standardized set of interference signals and characterization of their impact on its timekeeping capability. The performance of a particular receiver against interference signals is out of the scope of this research work. Hence without loss of generality, throughout this document, the experimental analysis is based on performance of a single timing-grade receiver. It is obvious that, the same methodology can be applied to other timing-grade receivers.

The rest of this document is organized as follows: In Section II we discuss different categories of GNSS interference signals we used to test the timing-grade receiver against. In Section III experimental methods tailored towards testing of timing-grade receivers against GNSS interference signals are presented. In Section IV theoretical background for assessment of timing-grade receivers is explained. Experimental results are provided in Section $\mathrm{V}$ and finally conclusions are drawn in Section VI.

\section{THREAT CLASSIFICATION}

One of the major outcomes of the STRIKE3 project was to identify 5 types of signals which were observed to be the most common categories of interference signals encountered in real world scenario. The idea behind categorizing GNSS interference types was not to propose a fixed set of threats to cover all types of interference signals, but instead to develop draft standards for testing receivers against interference signals which have been observed in the real world through a dedicated interference monitoring network. Using real interference threats which were detected in the field enables 
interested parties (e.g. certification bodies, application developers, receiver manufacturers, etc.) to better assess the risk to GNSS performance during operations, and to develop appropriate counter-measures. However, with many thousands of potential threats being detected by the monitoring network, it is impractical to test receivers against all detected threats. Therefore, an initial selection process was done, and a baseline set of 5 types of threats were selected for inclusion in the draft test standard for receiver testing [8]. 5 types of interference threats, as described in Table 1 are wide swept frequency with fast repeat rate, multiple narrow band signals, triangular and triangular wave swept frequency, and tick swept frequency. The interference threat templates are proprietary information, and thus no further information (e.g. frequency domain representation) can be disclosed in this document, however according to the frequency domain representations of each of the threat templates in Table 1, their bandwidth is close to $10 \mathrm{MHz}$ and they interfere with GPS L1 frequency band.

\section{EXPERIMENTAL METHODOLOGY}

In order to come to a qualitative conclusion on effects of interference on timing-grade GNSS receivers, we performed multiple tests where we measured the timing solution of the receiver under test (RUT) against a reference clock. Section A starts off with the hardware setup of the measurement platform after which Section B discusses the test scenario in detail.

\section{A. Instrumentation}

Fig. 1 shows the measurement platform. The clean GNSS signal is generated by a multi-constellation, multi-frequency Spectracom GSG-6 hardware simulator (GSG) [11]. RF interference signals based on the threats discussed in Section II were used as templates. We generated the interference signals by a Keysight vector signal generator N5172B (VSG) [12] which was configured to replay the templates. An Agilent universal time/frequency counter 53230A (TIC) [13] measured the time elapsed between the rising edge of the pulse per second (PPS) signal generated by the GSG and the rising edge of the PPS signal generated by RUT. A $10 \mathrm{MHz}$ sinusoidal signal obtained from live GNSS signals and a Septentrio PolaRx5S professional-grade receiver was distributed to the VSG, TIC, GSG and RUT in daisy-chain mode [15]. The RUT was configured to use GPS L1 C/A and Galileo E1 signals whereas the Septentrio PolaRx5S received GPS (L1P, L1 C/A, L2, L5), GLONASS (L1, L2, L3), Galileo (E1, E5ab, AltBoc, E6), SBAS (L1, L5).

The RUT, TIC, VSG and GSG were configured to use the $10 \mathrm{MHz}$ sinusoidal signal as input to their local oscillator. This ensures a consistent testing framework. RUT's mode of functionality was set to position-hold mode and was configured to generate PPS signal with $1 \mathrm{~Hz}$ repetition rate either if it had a position fix or if it was operating in holdover mode. On the other hand, the GSG was configured to generate PPS signal for the entire duration of the experiment. The GSG was configured to generate a PPS signal with a duty-cycle of $1 / 10$ (i.e. $1 / 10$ high and 9/10 low) whereas the RUT was configured to generate a PPS signal with a duty-cycle of 5/95. We collected measurements performed by the TIC and NMEA messages as reported by the RUT simultaneously with 1 second periodicity. These measurements were then analyzed in post-processing using a software script.

\section{B. Experiment Scenarios}

The RUT was subjected sequentially to the different threat templates identified in Section II. Provisions were taken to ensure that the test environment was uniform for all the test cases. These include length of the coaxial cables, duration of

Table 1. Characteristics of the interference templates.

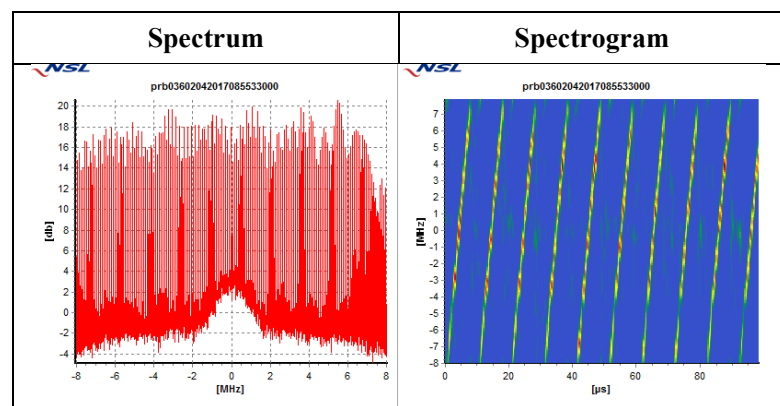

(a) wide swept frequency with fast repeat rate
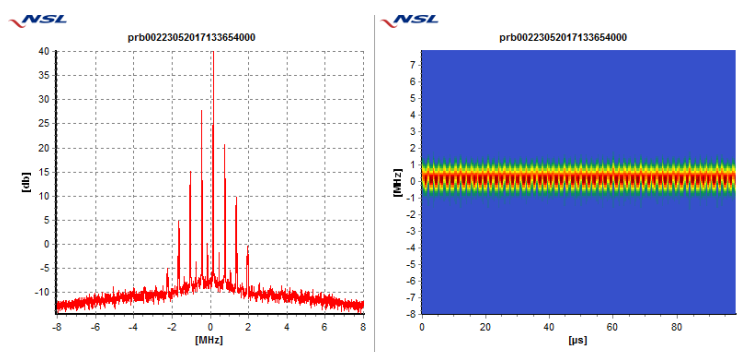

(b) multiple narrow band signals
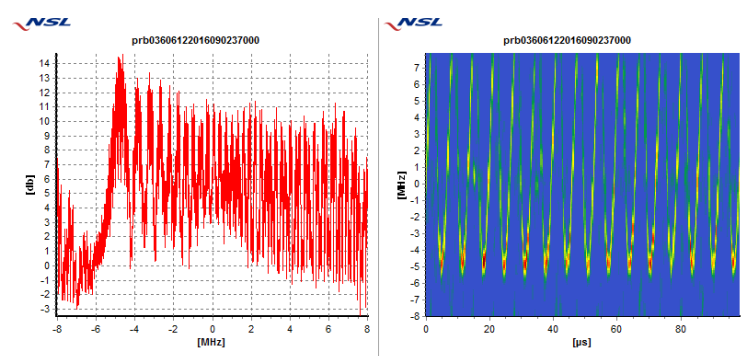

(c) triangular

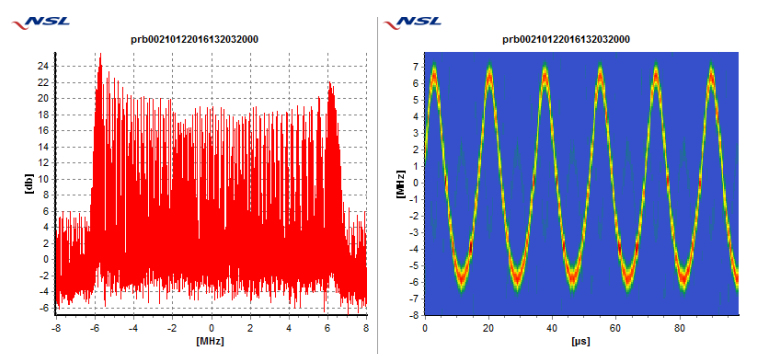

(d) triangular wave swept frequency
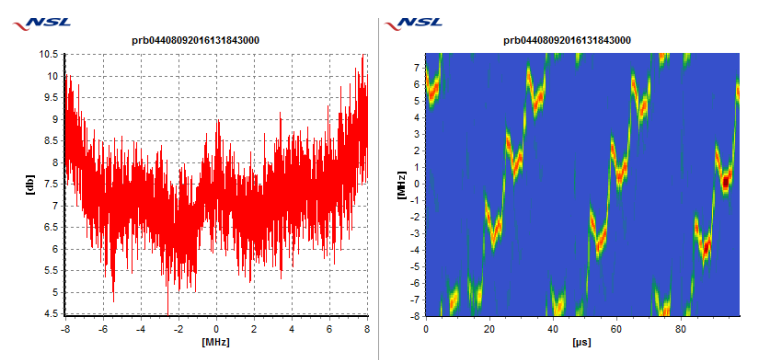

(e) tick swept frequency 


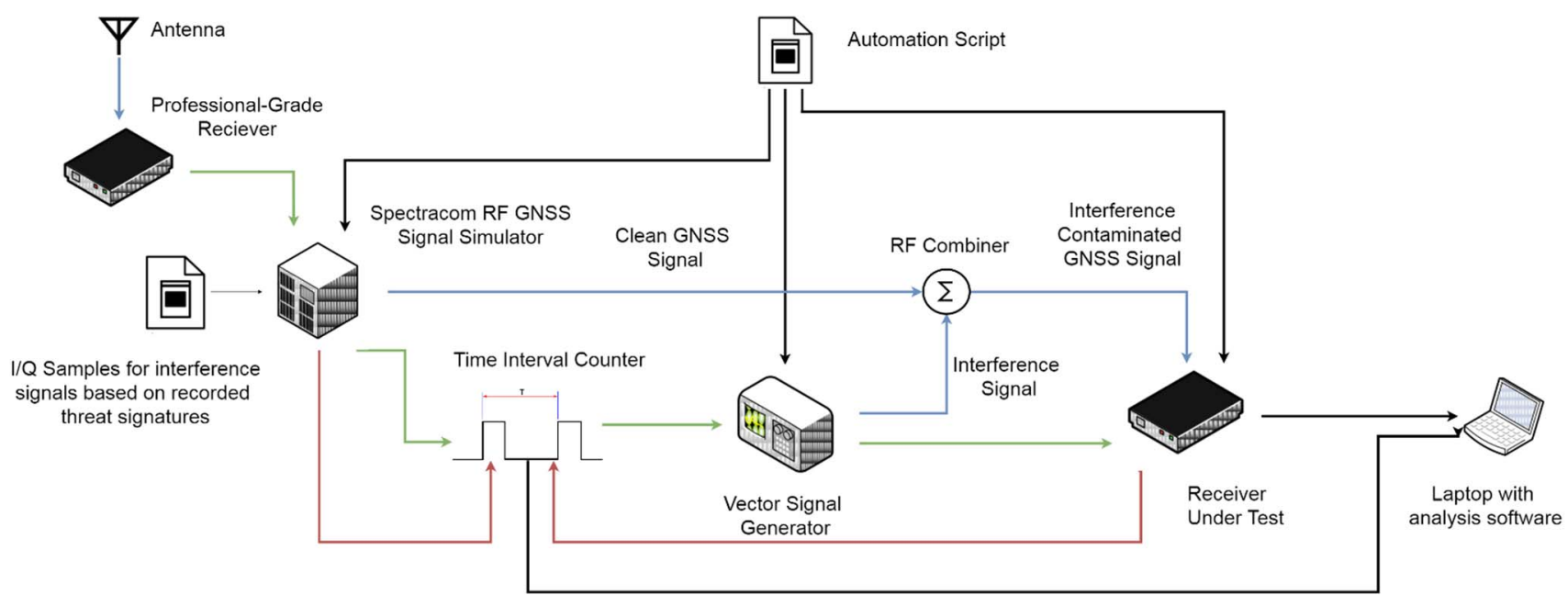

Fig 1. Test platform. Red directional lines denote PPS signal whereas blue directional lines indicate RF signal flow and green directional lines show the distribution of $10 \mathrm{MHz}$ sinusoidal signal.

the tests, initial operating state of the instruments, etc. Decisive factors in devising the test scenarios were:

- Mathematical tools described in Section IV require long duration of collected data for validity of the results [15].

- GSG, VSG, TIC must stabilize to their nominal working conditions before scenario starts.

- RUT must stabilize to its nominal working condition before being subjected to the threat signals.

- Power of the interference signals varies with time.

The chosen test scenario is depicted in Fig. 2. Throughout the whole test session, the GSG was transmitting clean GNSS signals with a set of configuration parameters as specified in Table 2, whereas the jamming signal as transmitted by the VSG was introduced only 5 hours after the test case started. The Jamming signal at this point was configured so that jamming to signal power ratio $(\mathrm{J} / \mathrm{S})$ was $5 \mathrm{~dB}$ and it lasted for 10 minutes. After every 10 minutes, $\mathrm{J} / \mathrm{S}$ was increased by 5 $\mathrm{dB}$ till it reached the maximum $\mathrm{J} / \mathrm{S}$ of $65 \mathrm{~dB}$ which again was planned to last for 10 minutes. After this point, a new sequence began in which the $\mathrm{J} / \mathrm{S}$ was reduced by $5 \mathrm{~dB}$ after every 10 minutes. This sequence terminated with $\mathrm{J} / \mathrm{S}$ of $0 \mathrm{~dB}$ which lasted for 10 minutes. The same sequence was repeated for the second time. The rest of the test scenario did not involve jamming of the RUT. The total duration of the test scenario was 24 hours.

We performed 5 tests with signals representing jamming templates presented in Section II namely, chirp wide sweep with a fast repeat rate, multiple narrow band signals, triangular chirp, triangular chirp wave and chirp tick. In addition, since

Table 2. Simulated scenario configuration

\begin{tabular}{|l|l|}
\hline Constellation & GPS / Galileo \\
\hline Number of satellites & $10(\mathrm{GPS}) / 10($ Galileo $)$ \\
\hline Broadcast signal & $\mathrm{L} 1 \mathrm{C} / \mathrm{A} / \mathrm{E} 1$ \\
\hline Ionosphere model & Klobuchar \\
\hline Troposphere model & Saatamoinen \\
\hline Scenario start time & 01.02 .2018 12:00:00 UTC \\
\hline Scenario duration & 24 hours \\
\hline
\end{tabular}

the RUT under nominal conditions is subject to imperfections, we performed a baseline test which did not involve jamming of the RUT at all. By applying the mathematical tools introduced in Section IV, we can characterize the RUT as a clock and compare its accuracy and stability under different interference conditions to the baseline case.

\section{KEY PERFORMANCE INDICATORS (KPI)}

In this section we point out the mathematical tools used for assessing the performance of the RUT. The tools used in this document for such a characterization are based on those defined in [15] for primary reference clocks (PRC). Two main specifications of time measurement are accuracy and stability [16].

\section{A. Accuracy}

As is dictated by the test platform in Fig. 1 accuracy of the RUT as a PRC at each time instance can be expressed as [15]:

$$
\delta t=t_{R U T}-t_{R E F}
$$

Where $t_{R U T}$ represents the measured on-time pulse as generated by the RUT and $t_{R E F}$ represents the reference ontime pulse.

\section{B. Stability}

Two quantities often used to characterize the stability of clocks in telecommunication systems are the maximum time interval error (MTIE) and time deviation (TDEV) [15], [16] and [17]. Given a sequence $\left\{\delta t_{i}\right\}$ for $i \in[1, N]$ with no dead time (dead time refers to the time between time-ordered data sets when no measurement of $\left(\delta t_{i}\right)$ is taken) between adjacent $\left(t_{i}\right)$ with sampling time $\tau_{0}$, MTIE can be calculated as follows [18]:

$$
\operatorname{MTIE}(\tau)=\max _{1 \leq k \leq N-n}\left(\max _{k \leq i \leq k+n} \delta t_{i}-\min _{k \leq i \leq k+n} \delta t_{i}\right)
$$

Where $n=1,2, \ldots, N-1$ denotes the number of measurements within the interval of analysis $\tau=n \tau_{0}$. Alternatively, instability of a clock in time domain can be defined by TDEV. TDEV as a function of an averaging time $\tau$ can be estimated as: 


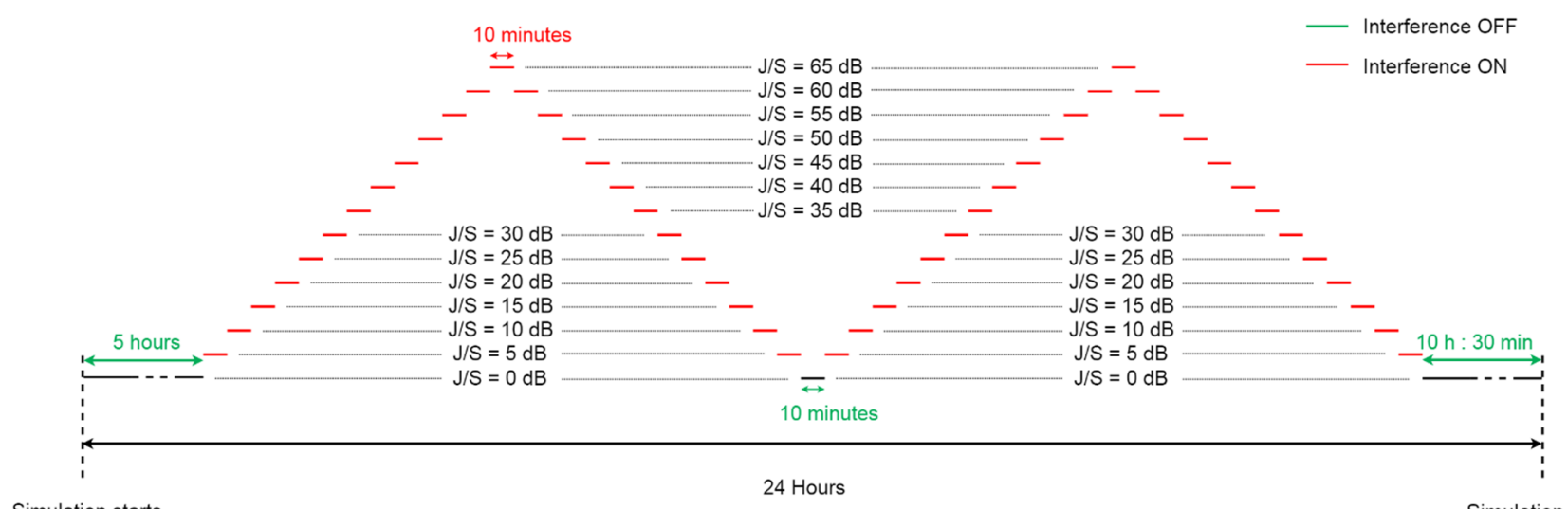

Simulation starts
Fig 2 . Test scenario. J/S denotes jamming to signal power ratio at the front-end of the RUT.

Similar to the baseline test case, for the test cases during

$\sigma_{\delta t}(\tau) \cong$

$\tau / \sqrt{3}\left\{\frac{\sum_{j=1}^{N-3 n+1}\left[\sum_{i=j}^{n+j-1}\left(\delta t_{i+2 n}-2 \delta t_{i+n}+\delta t_{i}\right)\right]^{2}}{2 \tau^{2} n^{2}(N-3 n+1)}\right\}^{1 / 2}$

Like MTIE, this variance assumes no dead time between adjacent $\left(\delta t_{i}\right)$ measurements. In [15] requirements for a PRC are specified in terms of maximum allowed values of MTIE and TDEV as calculated by (2) and (3). These requirements are listed in Tables. 3,4.

\section{EXPERIMENTAL RESULTS}

In this section we discuss the results of the experiments as outlined in Section III. For the data collected from each experiment, we calculated the KPIs which were introduced in Section IV. We calculated the accuracy of the RUT using (1) where $t_{R E F}$ is considered on-time pulse measurements as generated by the GSG. This is a valid argument since under nominal conditions, the RUT measures its solution based on the signals generated by the GSG, and on the other hand the PPS signal generated by the GSG reflects its time evolution. Doing so for all the experiments ensures a consistent outcome.

Accuracy and stability of the RUT under nominal conditions were calculated from the data collected from the baseline test. Fig. 3 shows the accuracy and stability values for the baseline test. The TDEV values on a logarithmic scale as shown in Fig. 3 provide the tool for characterizing random behavior of the RUT whereas MTIE in the same figure is a measure of its stability over time. According to both TDEV and MTIE values shown in Fig. 3, the RUT during baseline test satisfies the requirements for a PRC.

Table 3.

\begin{tabular}{|ccc|}
\hline ITU limits for MTIE as a function of analysis interval $\tau$ \\
\hline $0.275 \times 10^{-3} \tau+0.025 \mu \mathrm{s}$ & for & $0.1<\tau<1000 \mathrm{~s}$ \\
$10^{-5} \tau+0.29 \mu \mathrm{s}$ & for & $\tau>1000 \mathrm{~s}$ \\
\hline
\end{tabular}

Table 4.

\begin{tabular}{|ccc|}
\hline \multicolumn{3}{|c|}{ ITU limits for TDEV as a function of analysis interval $\tau$} \\
\hline $3 n s$ & for & $0.1<\tau<100 \mathrm{~s}$ \\
$0.03 \tau \mathrm{ns}$ & for & $100<\tau<1000 \mathrm{~s}$ \\
$30 \mathrm{~ns}$ & for & $1000<\tau<10000 \mathrm{~s}$ \\
\hline
\end{tabular}
which the RUT was subjected to different jamming signals, accuracy and stability values were calculated. Fig. 4 shows the values of the accuracy of the RUT over 24 hours corresponding to 5 representative jamming signals. Time offset values of the RUT as shown in Fig. 4 for all the test sessions oscillate around 124 nanoseconds for the first 5 hours. After introducing the interference signals at this point,

(a)

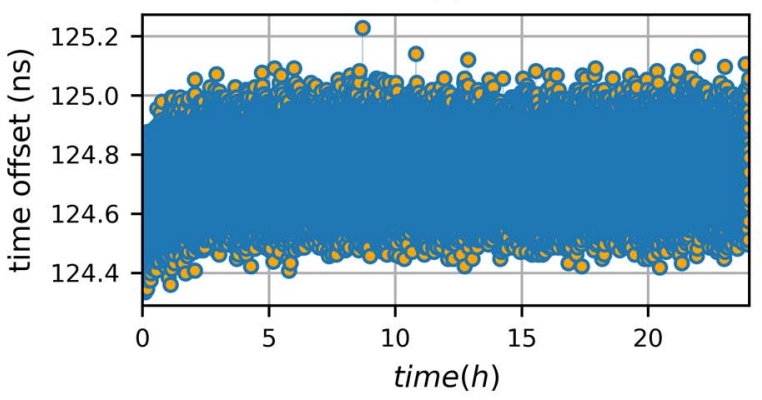

(b)

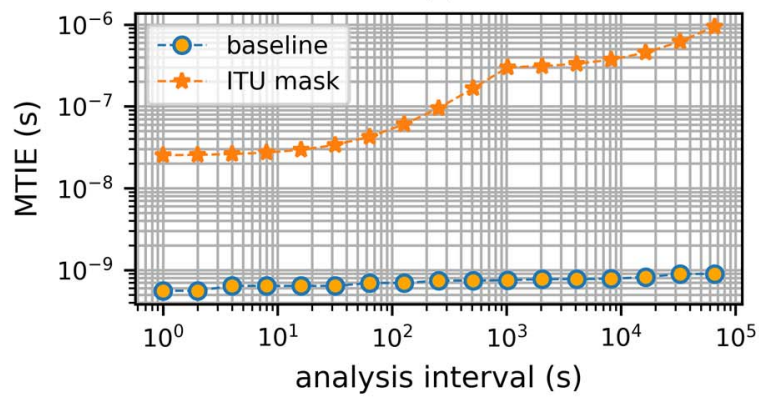

(c)

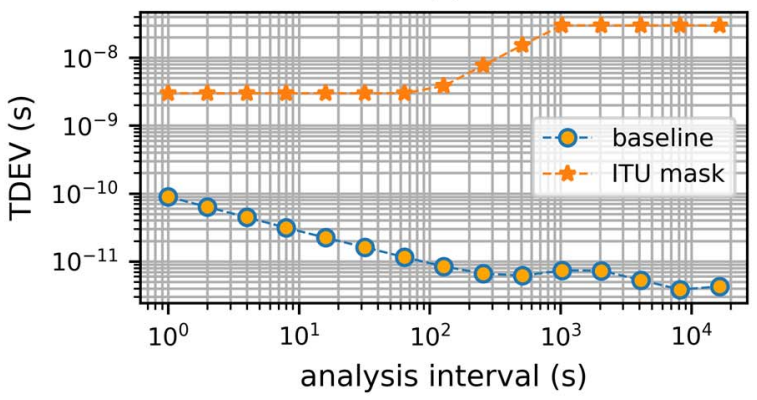

Fig 3. Results of the baseline test. Figure (a) shows the accuracy of the RUT whereas figures (b) and (c) show its' MTIE and TDEV values. 
(a)

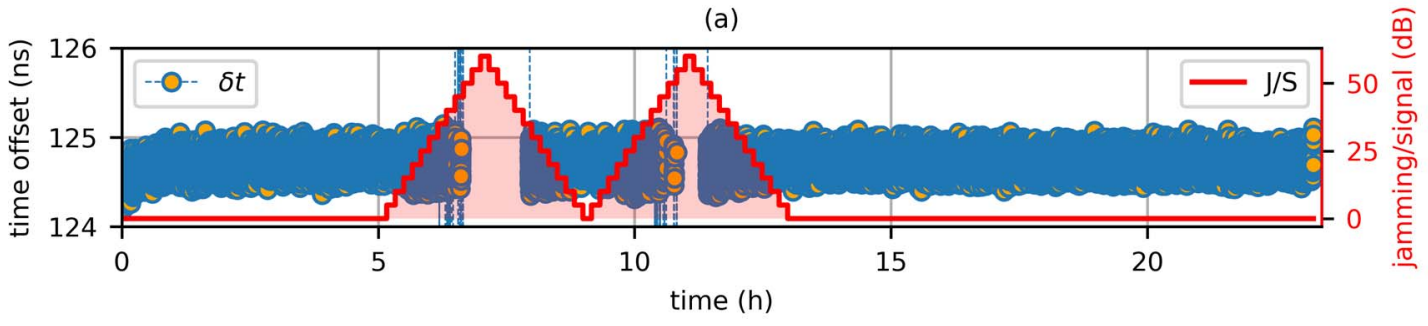

(b)

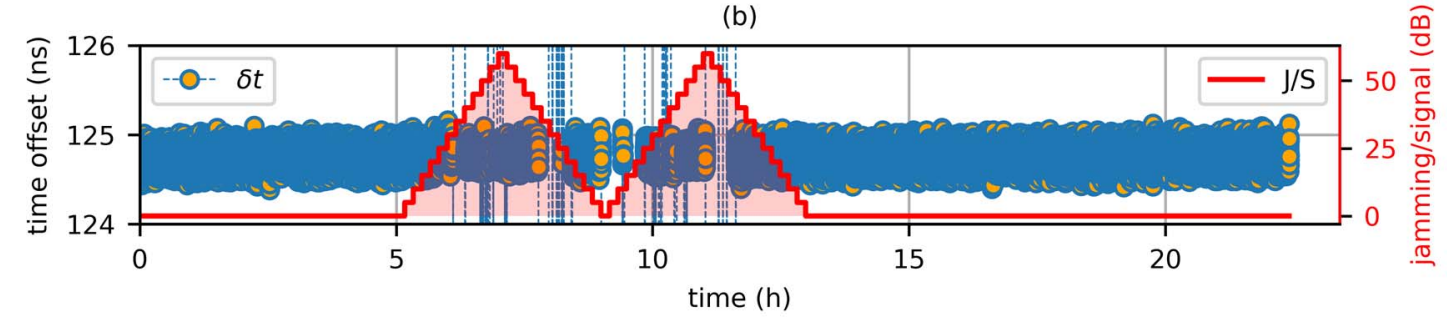

(c)

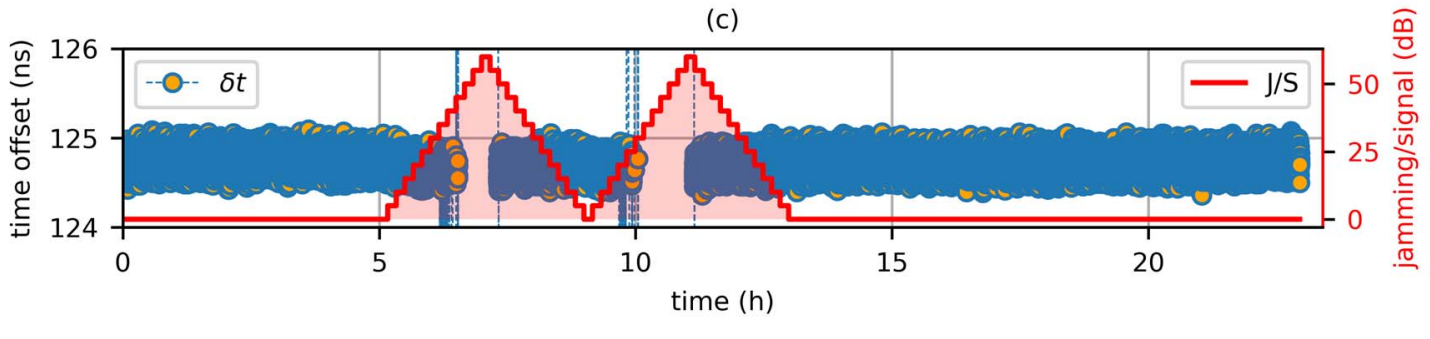

(d)

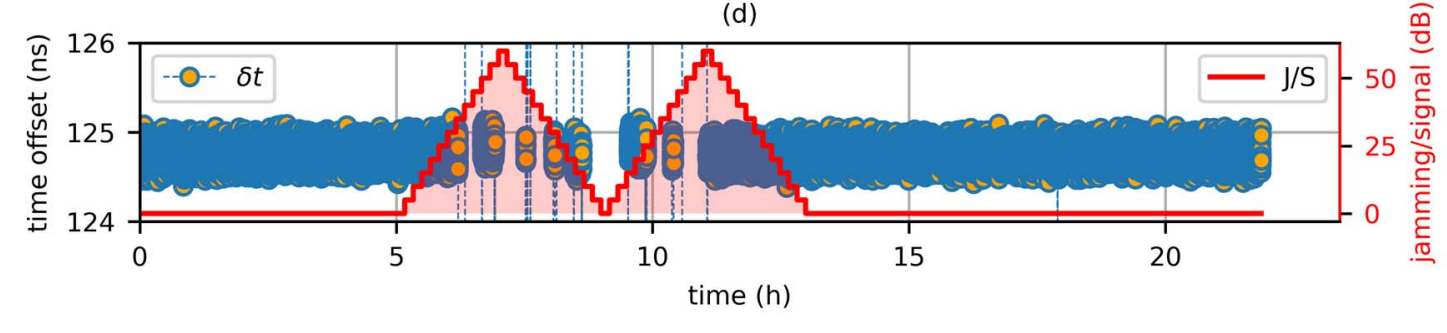

(e)

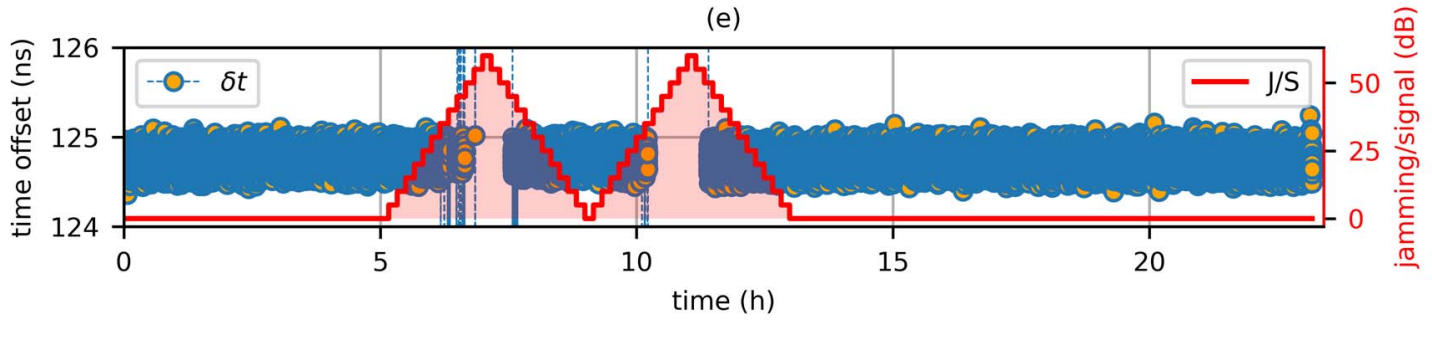

Fig 4. Time offset measurements for test cases in which the RUT was subjected to interference signals. Figures (a), (b), (c), (d) and (e) correspond to jamming templates of wide swept frequency with fast repeat rate, multiple narrow band signals, triangular and triangular wave swept frequency respectively. The red lines show the $\mathrm{J} / \mathrm{S}$ of the interference signals in $\mathrm{dB}$.

and gradually increasing the $\mathrm{J} / \mathrm{S}$ power, time offset values show a different behavior. This behavior differs across different test sessions but as it will be discussed later in this section, some of the interference signals managed to force the RUT to lose the position fix. Consequently, the RUT continues generating the PPS signal in holdover mode, based only on its internal oscillator for some finite time. During this time, the clock of the RUT drifts. This drift is reflected on the time offset values. For instance, according to Fig. 4(a), the RUT lost the position fix at 24002 seconds after the start of the test session which corresponds to $\mathrm{J} / \mathrm{S}$ of $55 \mathrm{~dB}$ but it continued to generate the PPS signal for the next 10 seconds. During this time, the time offset values grew in magnitude until the RUT stopped generating the PPS signal. After this point in time, the TIC did not log any measurements until the $\mathrm{J} / \mathrm{S}$ was small enough for the RUT to regain the position fix. On the contrary, in some cases, the interference signal could not force the RUT to lose the position fix, but it continued generating the PPS signal with a degraded accuracy and stability figures. For example, in case, the RUT was subjected to multiple narrow band interference signal, the average carrier to noise ratio (cno) of the satellites, which were used in 


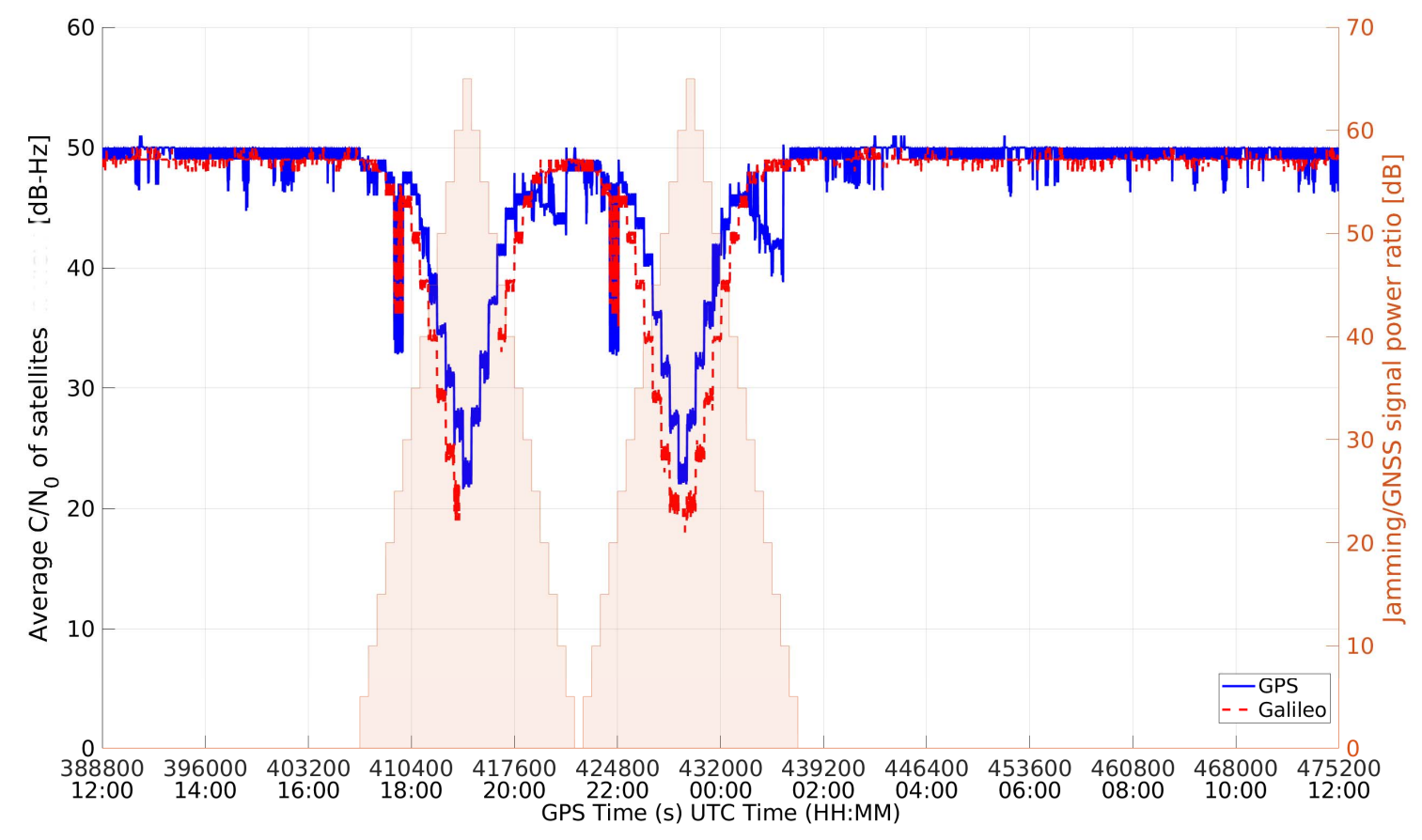

Fig 5. Average cno of the satellites tracked by the RUT under the influence of interference signal representing multiple narrow band. signals

the computation of the PVT solution by the RUT, is shown in Fig. 5. The average cno values drop as $\mathrm{J} / \mathrm{S}$ increases. Meanwhile, as can be seen from Fig. 4(b), the accuracy and stability values associated with the time offset values degrade as the $\mathrm{J} / \mathrm{S}$ increases.

Due to our specific test setup, the measurements as reported by the TIC were not time-tagged over the test scenario. Therefore, if a measurement of $\left(\delta t_{i}\right)$ was lost, calculation of the stability metrics for the whole data set using (2) and (3) would result in false values, since they assume no dead time (Section V). Sequel of this section describes a method to identify a fraction of the $\left\{\delta t_{i}\right\}$ sequence which does not contain any dead times.

The TIC in Fig. 1 was configured to perform a measurement of time offset $\left(\delta t_{i}\right)$ every one second, if the rising edge of the PPS signal generated by the GSG at the time instance $\left(t_{i}\right)$ occurred before that of the PPS signal generated by the RUT at the time instance $\left(t_{i}+\delta t_{i}\right)$ where $\left(t_{i}\right)$ and $\left(t_{i}+\delta t_{i}\right)$ are time coordinates defined with respect to the clock of the GSG. However if due to the jamming signals, the rising edge of the PPS signal generated by the RUT occurred before that of the PPS signal generated by the GSG, the TIC would not perform a measurement for the time instance $\left(t_{i}\right)$ but rather for $\left(t_{i+1}\right)$ only if the RUT generated a PPS signal at $\left(t_{i+1}+\delta t_{i+1}\right)$. Therefore, in this case, the time offset as measured by the TIC for $\left(t_{i}\right)$ would be missing and for $\left(t_{i+1}\right)$ would be equal to: $\left(t_{i+1}+\delta t_{i+1}-t_{i}\right)$.

NMEA messages reported by the RUT, include a position fix indicator sentence. Therefore, it is possible to correspond a time tag on time offset measurements for which the RUT held a GNSS fix if no dead time has previously occurred during the test session. On the other hand, if the RUT lost the position fix at some time instance $\left(t_{i}\right)$, the corresponding time offset measurement would be missing. Thus, a fraction of the $\left\{\delta t_{i}\right\}$ sequence which does not contain any dead time is equivalent to the earliest fraction of $\left\{\delta t_{i}\right\}$ measurements over which the RUT provides a position fix continuously and time offset measurements are smaller than 1 second. It is obvious that a fraction of time offset measurements which does not contain any dead times can be obtained as the smallest of the $\left(t_{i}\right) \mathrm{s}$ at which the RUT lost the position fix for the first time

Table 5 .

\begin{tabular}{|l|c|c|c|c|}
\hline \multicolumn{1}{|c|}{ Threat template } & $\begin{array}{c}\text { Time at which the } \\
\text { RUT lost position fix } \\
\text { for the first time }\end{array}$ & $\begin{array}{c}\text { J/S on which the RUT } \\
\text { lost position fix for the } \\
\text { first time }\end{array}$ & $\begin{array}{c}\text { Time at which } \boldsymbol{\delta} \boldsymbol{t}_{\boldsymbol{i}}>\mathbf{1} \\
\text { for the first time }\end{array}$ & $\begin{array}{c}\text { Number of lost time } \\
\text { offset measurements }\end{array}$ \\
\hline $\begin{array}{l}\text { wide swept frequency with } \\
\text { fast repeat rate }\end{array}$ & $24002(\mathrm{~s})$ & $55 \mathrm{~dB}$ & 23721 & 2728 \\
\hline $\begin{array}{l}\text { multiple narrow band } \\
\text { signals }\end{array}$ & N/A & N/A & 22000 & 5698 \\
\hline triangular & $23419(\mathrm{~s})$ & $45 \mathrm{~dB}$ & 23551 & 3762 \\
\hline $\begin{array}{l}\text { triangular wave swept } \\
\text { frequency }\end{array}$ & N/A & N/A & 22834 & 7664 \\
\hline tick swept frequency & $23405(\mathrm{~s})$ & $45 \mathrm{~dB}$ & 23580 & 2960 \\
\hline
\end{tabular}




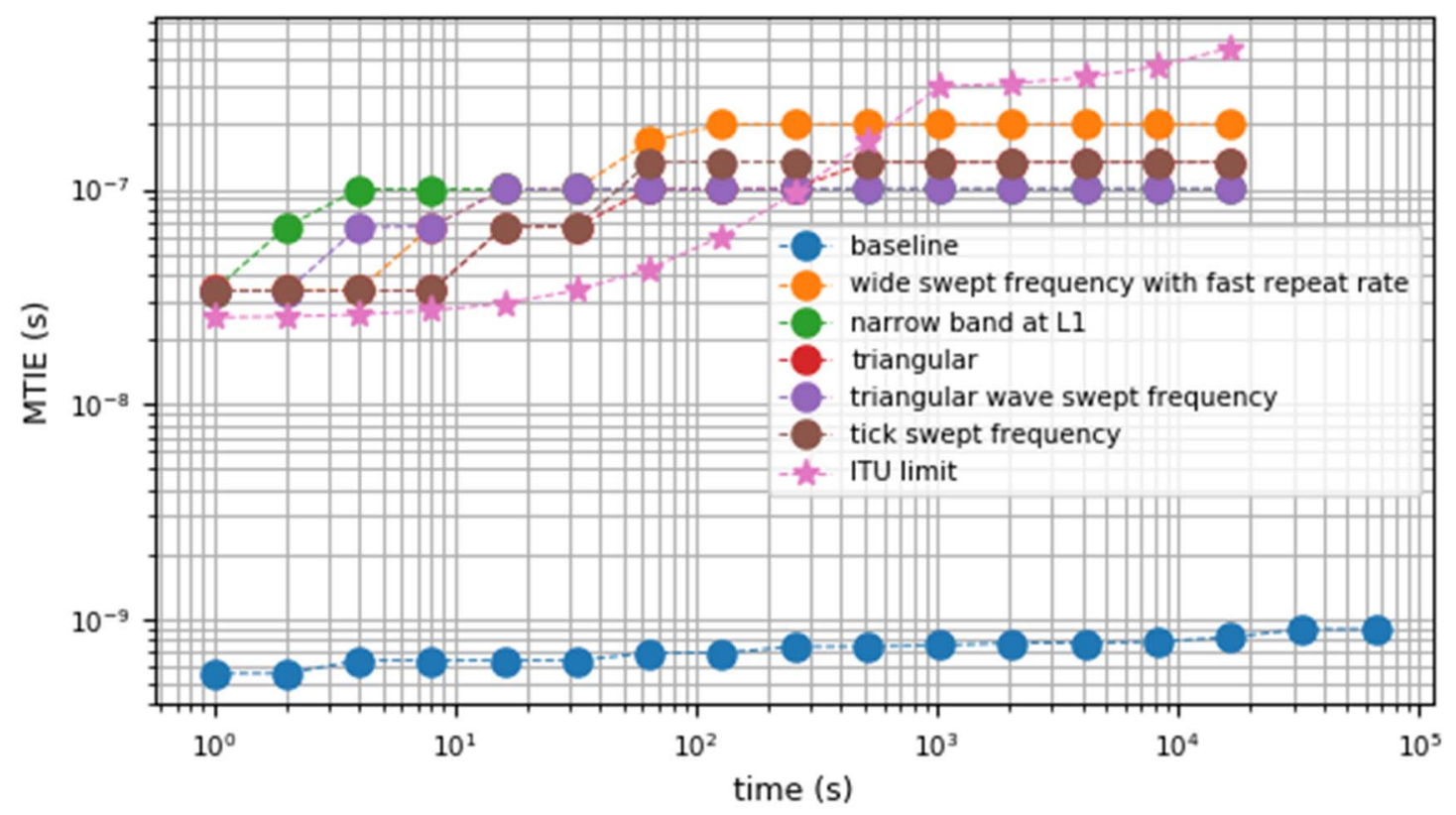

Fig 6. MTIE values.

and that at which $\delta t_{i}>1$ for the first time during a test session.

Logged NMEA messages show that the threat templates representing wide swept frequency with fast repeat rate, triangular and tick swept frequency forced the RUT to lose GNSS fix with $\mathrm{J} / \mathrm{S}$ of $55 \mathrm{~dB}, 45 \mathrm{~dB}$ and $45 \mathrm{~dB}$ respectively, whereas the RUT in presence of threat templates representing very narrow band signal and triangular wave swept frequency managed to retain the position fix over the whole test duration. Table 5 lists the number of lost time offset measurements as well as $\mathrm{J} / \mathrm{S}$ in decibels at the epoch which the RUT lost the position fix for the first time during the test scenario along with the corresponding time instance. It also lists the time instances at which the rising edge of the PPS signal generated by the RUT occurred before that of the PPS signal generated by the GSG for the first time for all the test cases.

MTIE and TDEV were calculated for the measurements performed for all of the test cases. In case of the baseline test, complete set of time offset measurements (i.e. 24 hours) were used whereas for test cases in which the RUT was jammed, only a fraction of the measurements with no dead time was used.

According to (4), accuracy of approximating TDEV is in direct relation with the length of the measurement set. On the other hand, due to presence of dead time in measurements performed for the test cases which involved jamming signals, only a smaller measurement set can be used to approximate the TDEV. Since the final measurement set is not long enough to approximate the TDEV values, without loss of generality, analysis are based on MTIE values alone. The results are depicted in Fig. 6.

Requirements as stated by the ITU dictate that a clock may be considered as a PRC only if its corresponding TDEV and MTIE values remain below a defined limit (Table 3, 4). Results in Fig. 6 show that in case of the baseline test, the RUT can be considered as a PRC since the corresponding MTIE values remain below the limit defined by the ITU whereas the
RUT in presence of any of the interference signals violated ITU requirements for a PRC.

\section{CONCLUSIONS}

We used established standard GNSS receiver testing procedures and designed a test bed to evaluate the behavior of timing-grade GNSS receivers once subjected to interference signals. We introduced interference signals which are replicas of the most common categories of interference signals encountered in real world scenario. These consist of wide swept frequency with fast repeat rate, multiple narrow band signals, triangular and triangular wave swept frequency, and tick swept frequency which were identified within STRIKE3 project. The GNSS receiver was subjected to the interference signals over 24 hours and multiple measurements were performed. Measurements consist of time offset values of the time solution compared to a reference clock and NMEA messages written out by the receiver under threat. In postprocessing, the stability and accuracy measures of the receiver were characterized for each test case. To do so, time deviation and maximum time interval error values were calculated, but it was shown that only the latter produce valid figures. The results show that the receiver while subjected to healthy GNSS signals can be considered as a primary reference clock, whereas in presence of the interference signals representing wide swept frequency with fast repeat rate, multiple narrow band signals, triangular and triangular wave swept frequency, and tick swept frequency templates violate the ITU requirements and can no longer be used as a primary reference clock.

Therefore, this paper validates the testing procedures which were standardized by the STRIKE3 project for testing of GNSS receivers against interference signals and provides a template for performing similar testing and validation exercises on timing-grade GNSS receivers. 


\section{ACKNOWLEDGMENT}

This work has been co-funded by the EU H2020 project entitled "Standardization of GNSS Threat reporting and Receiver testing through International Knowledge Exchange, Experimentation and Exploitation (STRIKE3)". The authors would like to acknowledge the STRIKE3 consortium for their constructive remarks on the test campaign and the results analysis of different receiver testing.

\section{REFERENCES}

[1] M. A. Lombardi et al, "Time and frequency measurements using the Global Positioning System", Cal Lab: International Journal of Metrology, vol. 8, no.3, pp. 26-31, 2001.

[2] L. Gasparini et al, "A digital circuit for jitter reduction of GPSdisciplined 1-pps synchronization signals", IEEE International Workshop on Advanced Methods for Uncertainty Estimation in Measurements, 2007, pp. 84-88.

[3] M. A. Lombardi, "The use of GPS disciplined oscillators as primary frequency standards for calibration and metrology laboratories ", NCSLI Measure, vol. 3, no. 3, 2008, pp.56-65.

[4] "Timing Scenario Set”, Spirent Communications Inc, 2019 [online] Available:https://www.spirent.com/media/datasheets/positioning/testpack-timing-scenario-set.pdf?la=en

[5] "Technology Anti-Jamming Performance of FURUNO Multi-GNSS timing receivers", [online]. Available: https://www.furuno.com/en/gnss/technical/anti_jamming_timing\#sec $\underline{1}$

[6] J. B. Bullock, "Test Results and Analysis of a Low Cost Core GPS Receiver for Time Transfer Applications", IEEE International Frequency Control Symposium, 1997.

[7] V. Ogrizovic, et al "Testing GPS generated 1PPS against a rubidium standard", ACTA IMEKO, Vol. 2, No. 1, 7-11, August 2013.

[8] STRIKE3 project deliverable, D4.2: Draft Standards for Receiver Testing against Threats. Version 2.0. Available online via http://www.gnss-strike3.eu/ (accessed on December 12, 2018).

[9] Bhuiyan, M. Z. H., Ferrara, N. G., Amin, H., Thombre, S., Pattinson, M., Dumville, M. (2019) "Impact Analysis of a Standardized GNSS Receiver Testing against Real-world Interferences Detected at live Monitoring Sites," Sensors special issue on "Interference, Robustness and Complementary Solutions for GNSS-Based Navigation for Aerial Vehicles', Sensors 2019, 19(6): 1276, DOI: https://doi.org/10.3390/s19061276.

[10] Thombre, S., Bhuiyan, M. Z. H., Eliardsson, P., Gabrielsson, B. Pattinson, M., Dumville, M., Fryganiotis, D., Hill, S., Manikundalam, V., Pölöskey, M., Lee, S., Ruotsalainen, L., Söderholm, S., Kuusniemi, H. (2017) "GNSS Threat Monitoring and Reporting: Past, Present, and a Proposed Future," Royal Institute of Navigation Journal, published online: $07 \quad$ December, 2017, DOI: https://doi.org/10.1017/S0373463317000911.

[11] "Spectracom GSG-5/6 product brief" Orolla USA, Inc., April 2017. [online] Available at: http://spectracom.com/documents/gsg-56-seriesadvanced-simulators-datasheet/attachment

[12] "Keysight EXG X-Series Signal Generators N5171B Analog \& N5172B Vector datasheet", December 2017. [online] Available at: https://literature.cdn.keysight.com/litweb/pdf/5991. 0039EN.pdf?id=2163727

[13] Keysight Technologies, "53230A Series RF/Universal Frequency Counters/Timers-datasheet"[online] Available at https://www.keysight.com/en/pd-1893420-pn-53230A/350-mhzuniversal-frequency-counter-timer-12-digits-s-20-ps?cc=FI\&lc=fin

[14] D. Doberstein "Fundamentals of GPS Receivers, A Hardware Approach" Springer, New York, Ny, 2012.

[15] "Timing characteristics of primary reference clocks, International Telecommunication Union recommendation ITU-T G8.11, Amendment 1", Apr. 2015 [online]. Available: https://www.itu.int/rec/T-REC-G.8.11/en

[16] M. A. Lombardi "Fundamentals of Time and Frequency" in The Mechatronics Handbook, R. H. Bishop, CRC Press, 2002.

[17] IEEE standards coordinating committee 27 on time and frequency "IEEE standard definitions of physical quantities for fundamental frequency and time metrology--random instabilities", September 2008.
[18] S. Bregni "Measurement of maximum time interval error for telecommunications clock stability characterization" IEEE Transactions on Instrumentation and Measurement, vol. 45, no. 6, October 1997. 